\title{
A Plasma Protease Which Is Expressed during Supramaximal Stimulation Causes In Vitro Subcellular Redistribution of Lysosomal Enzymes in Rat Exocrine Pancreas
}

\author{
M. Saluja, A. Saluja, M. M. Lerch, and M. L. Steer \\ Department of Surgery, Beth Israel Hospital and Harvard Medical School, \\ Harvard Digestive Diseases Center, Boston, Massachusetts 02215
}

\begin{abstract}
The complex events by which digestive enzyme zymogens and lysosomal hydrolases are segregated from each other and differentially transported to their respective membrane-bound intracellular organelles in the pancreas have been noted to be disturbed during the early stages of several models of experimental pancreatitis. As a result, lysosomal hydrolases such as cathepsin $B$ are redistributed to the subcellular zymogen granulerich fraction and lysosomal hydrolases as well as digestive enzyme zymogens are colocalized within large cytoplasmic vacuoles. The current study was designed to create an in vitro system that would reproduce this redistribution phenomenon. Our results indicate that cathepsin $B$ redistribution occurs when rat pancreatic fragments are incubated with a supramaximally stimulating concentration of the cholecystokinin analogue caerulein along with plasma from an animal subjected to in vivo supramaximal caerulein stimulation. Neither the plasma nor a supramaximally stimulating concentration of caerulein, alone, is sufficient to induce in vitro cathepsin $B$ redistribution. The ability of the plasma to induce in vitro cathepsin redistribution is dependent upon its content of a $10,000-30,000-D$ protein and is lost by exposure to protease inhibitors. In vitro cathepsin B redistribution also occurs when rat pancreatic fragments are incubated with plasma obtained from opossums with hemorrhagic necrotizing pancreatitis caused by bile/pancreatic duct ligation. (J. Clin. Invest. 1991.87:1280-1285.) Key words: pancreatitis • intracellular trafficking • lysosomes • cathepsin B
\end{abstract}

\section{Introduction}

We have recently shown that the early stages of two forms of experimental pancreatitis (diet-induced and secretagogue-induced) share the common attribute of colocalization of digestive enzyme zymogens with lysosomal hydrolases inside large cytoplasmic vacuoles. This conclusion was based on the results of morphological studies using immunolocalization techniques which demonstrated the presence of digestive zymogens as well as lysosomal hydrolases within the same organelle (1-3) and on the results of biochemical studies which used subcellu-

Address correspondence and reprint requests to Dr. Michael L. Steer, Dept. of Surgery, Beth Israel Hospital, 330 Brookline Ave., Boston, MA 02215 . 1990

Received for publication 2 May 1990 and in revised form 16 October

J. Clin. Invest.

(C) The American Society for Clinical Investigation, Inc.

$0021-9738 / 91 / 04 / 1280 / 06 \$ 2.00$

Volume 87, April 1991, 1280-1285 lar fractionation techniques to demonstrate redistribution of cathepsin B and other lysosomal enzymes from the lysosomeenriched to the zymogen granule-enriched fraction $(4,5)$. Subcellular redistribution and colocalization of cathepsin B with digestive zymogens was also noted to follow pancreatic duct obstruction (6). Because cathepsin B can activate trypsinogen (7) and trypsin can activate the other digestive zymogens, the colocalization of digestive enzyme zymogens with lysosomal hydrolases could lead to intracellular digestive enzyme activation and be an important triggering event in the development of acute pancreatitis (8).

The secretagogue-induced model of experimental pancreatitis involves infusing rats with a dose of the cholecystokinin analogue caerulein in excess of that required to cause a maximal rate of digestive enzyme secretion from the pancreas (9, 10). Within 3-4 h of beginning that infusion, hyperamylasemia and massive interstitial pancreatic edema occur (10), and the redistribution of lysosomal enzymes to the zymogen granuleenriched fraction can be demonstrated (4). In the current communication, we report the results of studies which demonstrate that the redistribution phenomenon can be reproduced in a completely in vitro system. They indicate that cathepsin B redistribution is dependent upon the presence of a supramaximally stimulating concentration of caerulein along with a plasma factor which is expressed in response to in vivo supramaximal caerulein stimulation. Cathepsin B redistribution is also reported to occur when rat pancreatic fragments are exposed, in vitro, to plasma obtained from opossums with duct obstruction-induced pancreatitis.

\section{Methods}

Preparation and incubation of pancreas fragments. The pancreas from 200-g male Wistar rats was harvested and cut into fragments measuring 1-2 $\mathrm{mm}^{3}$. The fragments were suspended in Hepes-Ringer buffer, $\mathrm{pH}$ 7.4 , containing $118 \mathrm{mM} \mathrm{NaCl}, 5 \mathrm{mM} \mathrm{KCl}, 1 \mathrm{mM} \mathrm{MgCl} 2,1.26 \mathrm{mM}$ $\mathrm{CaCl}_{2}, 10 \mathrm{mM}$ Hepes, $0.01 \%$ soyabean trypsin inhibitor, and $1 \% \mathrm{Ea}-$ gle's basal amino acids. When indicated, this buffer was augmented by addition of caerulein $(0.1 \mu \mathrm{M})$ and/or rat or opossum plasma $(80 \%$, $\mathrm{vol} / \mathrm{vol})$. Pancreas fragments were incubated at $37^{\circ} \mathrm{C}$ under an atmosphere of $95 \% \mathrm{O}_{2}-5 \% \mathrm{CO}_{2}$ for $4 \mathrm{~h}$ before subcellular fractionation. At the conclusion of each incubation, the $\mathrm{pH}$ was measured and consistently found to be $7.4 \pm 0.1$.

Subcellular fractionation. Pancreas fragments were washed twice in pH 7.0 buffer containing $5 \mathrm{mM}$ Mops, $1 \mathrm{mM} \mathrm{MgSO}_{4}$, and $250 \mathrm{mM}$ sucrose. After washing, they were homogenized by two up-and-down strokes of a motorized glass-teflon homogenizer. Unbroken cells and debris were removed by low-speed centrifugation $(150 \mathrm{~g} \times 10 \mathrm{~min}$, $4^{\circ} \mathrm{C}$ ). The resulting supernatant was considered to be the entire sample for later calculations and to contain $100 \%$ of all measured components. Subcellular fractionation was performed using differential centrifugation as described by Tartakoff and Jamieson (11) with minor modifica- 
tions which have been previously reported (6). Briefly, the supernatant described above was centrifuged $\left(1,000 \mathrm{~g} \times 15 \mathrm{~min}, 4^{\circ} \mathrm{C}\right)$ to yield a "zymogen granule" pellet and a supernatant. The latter was harvested and centrifuged $\left(12,000 \mathrm{~g} \times 12 \mathrm{~min}, 4^{\circ} \mathrm{C}\right)$ to obtain a "lysosomal" pellet and a supernatant. This supernatant was centrifuged $(105,000 \mathrm{~g}$ $\times 60 \mathrm{~min}, 4^{\circ} \mathrm{C}$ ) to obtain a "microsomal" pellet and postmicrosomal supernatant or "soluble" fraction. The various fractions were characterized by measuring markers (DNA, RNA, amylase, cathepsin B, cytochrome oxidase) as previously described (10).

Preparation of "caerulein-plasma". Male Wistar rats weighing $\sim 200 \mathrm{~g}$ were anesthetized with pentobarbitol $(1.5 \mathrm{mg} / \mathrm{rat}$ i.p. $)$ and a PE-10 cannula was placed in the external jugular vein. The animals were allowed to awaken and recover from the anesthetic overnight while the patency of the cannula was maintained by infusion of 150 $\mathrm{mM} \mathrm{NaCl}$ containing $30 \mathrm{U} / \mathrm{ml}$ heparin at a rate of $0.21 \mathrm{ml} / \mathrm{h}$. The next day, each animal was infused with a similar solution containing caerulein at a concentration calculated to deliver $5 \mu \mathrm{g} / \mathrm{kg}$ per $\mathrm{h}$. After infusion of this supramaximally stimulating dose of caerulein for $3.5 \mathrm{~h}$, the animals were sacrificed and plasma harvested for later use. All animals infused with this supramaximally stimulating dose of caerulein were observed to have grossly obvious pancreatic edema. For the most part, the plasma harvested from these caerulein-infused rats (termed "caerulein-plasma") was used within $48 \mathrm{~h}$ of caerulein infusion. Pilot experiments were performed which indicated that the effects of this plasma on the subcellular distribution of cathepsin B in pancreas fragments reported in this manuscript were not changed by the storage of the plasma, at $4^{\circ} \mathrm{C}$, for up to $1 \mathrm{wk}$.

Preparation of opossum pancreatitis plasma. Randomly trapped, healthy opossums (Didelphis virginiana) of either sex weighing 2.4-3.9 $\mathrm{kg}$ were fasted overnight and then anesthetized with an intraperitoneal injection of pentobarbital $(50 \mathrm{mg} / \mathrm{kg})$. Using sterile technique, a midline upper abdominal incision was made, the cystic duct ligated, and the common bile/pancreatic duct ligated adjacent to the wall of the duodenum. Jaundiced control animals underwent the same procedure, including cystic duct ligation, but the common bile duct was ligated on the liver side of the pancreas (i.e., proximal to its junction with the pancreatic duct). Thus, in the jaundiced control animals, pancreatic juice continued to flow into the duodenum. The abdominal incision was then closed and the animals maintained on food and water ad libitum for $7 \mathrm{~d}$. They were then sacrificed with a lethal dose of pentobarbital, blood harvested from the right ventricle, and the pancreas examined. All of the animals with bile/pancreatic duct ligation had gross morphologic evidence of pancreatitis including fat and parenchymal necrosis, pancreatic edema, and areas of intrapancreatic hemorrhage. The jaundiced control animals had no gross changes suggestive of pancreatitis. These observations are consistent with those reported by Senninger et al. (12). Serum amylase values were $1,655 \pm 774$ and $270 \pm 68 \mathrm{U} / \mathrm{ml}$ in the pancreatitis and jaundiced control animals, respectively. Pancreatic water content was $78 \pm 3$ and $70 \pm 1 \%$ in the pancreatitis and control animals, respectively. Serum samples were prepared immediately and stored frozen $\left(-20^{\circ} \mathrm{C}\right)$ until later use.

Deproteination and ultrafiltration of plasma. Caerulein plasma was deproteinated by either heat or alcohol denaturation. The former was accomplished by addition of boiling water to the plasma sample (25\% $\mathrm{vol} / \mathrm{vol}$ ), whereas the latter was achieved by addition of acidified ethanol $(40 \%, \mathrm{vol} / \mathrm{vol} ; \mathrm{pH} 4.0)$. In each case, the resulting protein precipitate was removed by centrifugation and the remaining supernatant was concentrated to the original volume by speed-vac. In the process, the ethanol was removed. Caerulein plasma was ultrafiltered using centiprep-10 and centiprep- 30 membranes which have molecular weight cutoffs of 10,000 and 30,000 , respectively.

Enzyme assays. Cathepsin B activity was measured fluorimetrically using CBZ-arginyl-arginine-B-napthylamide as the substrate as described by McDonald and Ellis (13) with minor modifications (4). Amylase, DNA, RNA, and cytochrome oxidase were evaluated as described previously (10).

Material. Rats were purchased from Charles River Breeding Laboratories, Inc., Wilmington, MA. Opossums were obtained from R-

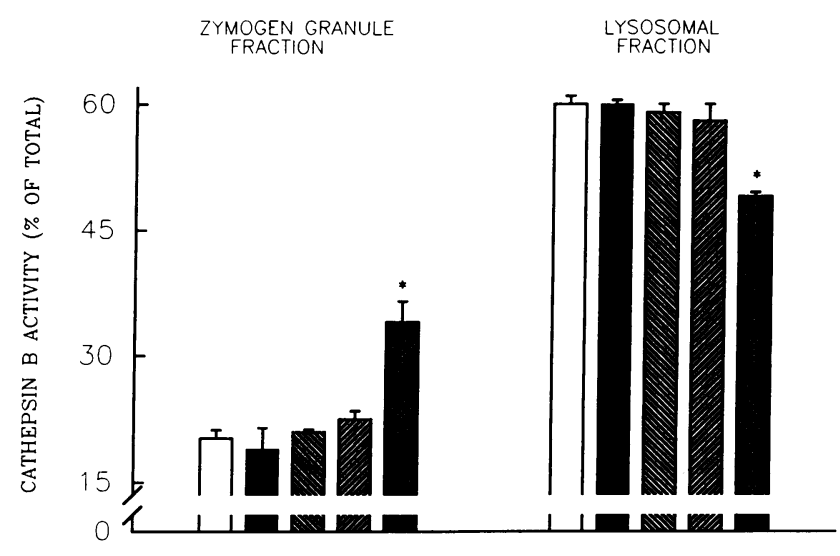

Figure 1. In vitro cathepsin-B redistribution. Pancreas fragments were incubated for $4 \mathrm{~h}$ at $37^{\circ} \mathrm{C}$ in buffer alone (open bars), buffer containing $0.1 \mu \mathrm{M}$ caerulein (solid bars), rat plasma from control animals (left diagonal bars), plasma from control animals plus $0.1 \mu \mathrm{M}$ caerulein (right diagonal bars), or plasma from rats infused with $5 \mu \mathrm{g} / \mathrm{kg}$ per $\mathrm{h}$ caerulein for $3.5 \mathrm{~h}$ to which $0.1 \mu \mathrm{M}$ caerulein was added (crosshatched bars). The fragments were then subcellularly fractionated and cathepsin B activity in the zymogen granule-enriched $(1,000 \mathrm{~g}$ $\times 10 \mathrm{~min})$ and lysosome-enriched $(12,000 \mathrm{~g} \times 10 \mathrm{~min})$ fractions measured. Bars denote mean, and vertical brackets denote SEM obtained from at least six separate experiments. *Value significantly different $(\alpha \leq 0.01)$ from other groups.

ZOO, Neshkoro, WI. Caerulein, Mops, PMSF, and Triton X-100 were from Sigma Chemical Co., St. Louis, MO. CBZ-arginyl-arginine-Bnapthylamide was obtained from Bachem. L-364,718 was the kind gift of Dr. R. Chang of Merck Sharp and Dohme Research Laboratories (West Point, PA). All other chemicals and reagents were of the highest purity available and from sources previously reported. The centiprep filters were purchased from Amicon Corp., Danvers, MA.

Analysis of data. The results reported in this paper represent the mean \pm SEM obtained from at least three separate experiments (with duplicated incubation in every experiment) for each observation. Error bars in the figures indicate SEM values, whereas the absence of these bars indicate that the SEM values were too small to illustrate. Analysis of variance was used to evaluate the significance of observed changes. If ANOVA indicated significant differences, then the means were evaluated by a multiple comparison method of Tukey (14) as described by Zar (15). The differences were considered significant if the $q$ value was more than the critical value when $\alpha \leq 0.05$.

\section{Results}

The subcellular distribution of cathepsin B in rat pancreatic fragments incubated in Hepes-Ringer buffer for $4 \mathrm{~h}$ is shown in Fig. 1. Addition of $0.1 \mu \mathrm{M}$ caerulein, ${ }^{1}$ plasma taken from a control animal, or a combination of control plasma plus 0.1 $\mu \mathrm{M}$ caerulein did not alter the subcellular distribution of cathepsin B (Fig. 1). In contrast, a significant change in the subcellular distribution of cathepsin B was noted when the fragments were incubated with $0.1 \mu \mathrm{M}$ caerulein combined with

${ }^{1}$ Preliminary experiments indicated that a maximal rate of amylase secretion from pancreas fragments occurred in the presence of $1 \mathrm{nM}$ caerulein and that higher concentrations caused inhibition of amylase secretion. The dose chosen for use in these in vitro studies, $0.1 \mu \mathrm{M}$, consistently caused inhibition of amylase secretion. Incubation of pancreas fragments with buffer containing higher concentrations of caerulein $(1 \mu \mathrm{M})$ also did not cause in vitro cathepsin B redistribution. 
plasma that had been harvested from rats previously infused with $5 \mu \mathrm{g} / \mathrm{kg}$ per h caerulein for $3.5 \mathrm{~h}$ ("caerulein plasma")(Fig. 1). Under those conditions, whereas cathepsin B total activity remained unchanged (not shown), the percent of cathepsin B activity in the zymogen granule-enriched fraction rose from $20.2 \pm 1.0$ to $34.0 \pm 2.5 \%$ and declined in the lysosome-enriched fraction from $60.0 \pm 1.5$ to $49.0 \pm 0.5 \%$. These changes are highly significant $(P<0.01)$. In contrast, the activity of cathepsin B in the microsomal and in the soluble (cytosolic) fractions was not altered by incubation in caerulein-plasma plus caerulein (data not shown). These findings indicate that in vitro incubation of pancreas fragments in either buffer or normal plasma containing a supramaximally stimulating concentration of caerulein does not cause subcellular redistribution of cathepsin B but that redistribution of cathepsin B from the so-called lysosomal fraction (12,000 $\mathrm{g} \times 10 \mathrm{~min}$ pellet) to a more dense fraction normally enriched in zymogen granules $(1,000 \mathrm{~g} \times 10 \mathrm{~min}$ pellet) occurs when pancreas fragments are incubated with a supramaximally stimulating concentration of caerulein along with plasma taken from rats which had been subjected to in vivo supramaximal caerulein stimulation.

The subsequent studies were undertaken to characterize the plasma factor(s) expressed after in vivo supramaximal caerulein stimulation which was found to permit in vitro subcellular redistribution of cathepsin $B$. The ability of caerulein-plasma to permit in vitro cathepsin B redistribution was found to be lost when the plasma was deproteinated either by heat or acidethanol denaturation (Table I). These observations indicate that the plasma factor expressed in response to in vivo caerulein infusion which permits in vitro cathepsin $B$ redistribution is a protein. In vitro redistribution of cathepsin B did not occur when pancreas fragments were incubated with caeruleinplasma to which the protease inhibitors phenylmethylsulfonyl fluoride (PMSF) and soyabean trypsin inhibitor had been added (Fig. 2). This finding indicates that in vitro cathepsin B

Table I. Characterization of the Plasma Factor Involved in Redistribution of Cathepsin B

\begin{tabular}{llc}
\hline & \multicolumn{2}{c}{ Cathepsin B activity (\% of total) } \\
\cline { 2 - 3 } \multicolumn{1}{c}{ Group } & ZG fraction & Lysosome fraction \\
\hline Buffer & $19.7 \pm 1.3$ & $60.2 \pm 1.3$ \\
CER plasma + CER & $29.5 \pm 0.8^{*}$ & $48.3 \pm 2.0^{*}$ \\
Alcohol denatured CER plasma & & \\
$\quad$ + CER & $17.3 \pm 2.5$ & $58.0 \pm 2.1$ \\
Heat denatured CER plasma + CER & $18.0 \pm 0.7$ & $63.0 \pm 2.3$ \\
CER plasma & $30.0 \pm 1.1^{*}$ & $50.0 \pm 1.5^{*}$ \\
CER plasma + L-364,718 & $18.3 \pm 2.1$ & $58.3 \pm 0.9$ \\
\hline
\end{tabular}

Rat pancreatic fragments were incubated in buffer, caerulein plasma (see legend for Fig. 1), deproteinated caerulein plasma or caeruleinplasma plus $0.1 \mathrm{mM} \mathrm{L-364,718}$. Caerulein-plasma was deproteinated either by precipitation with acidic ethanol or by adding the plasma to boiling water as described in the text. Caerulein (CER, $0.1 \mu \mathrm{M}$ final concentration) was added to some of the incubation mixtures as indicated. After $4 \mathrm{~h}$ of incubation at $37^{\circ} \mathrm{C}$, the percentage of total cathpesin B activity recovered in the zymogen granule-enriched and lysosome-enriched pellet was measured. Results shown are means \pm SEMs obtained from at least three separate experiments. * Significant differences compared with other groups.

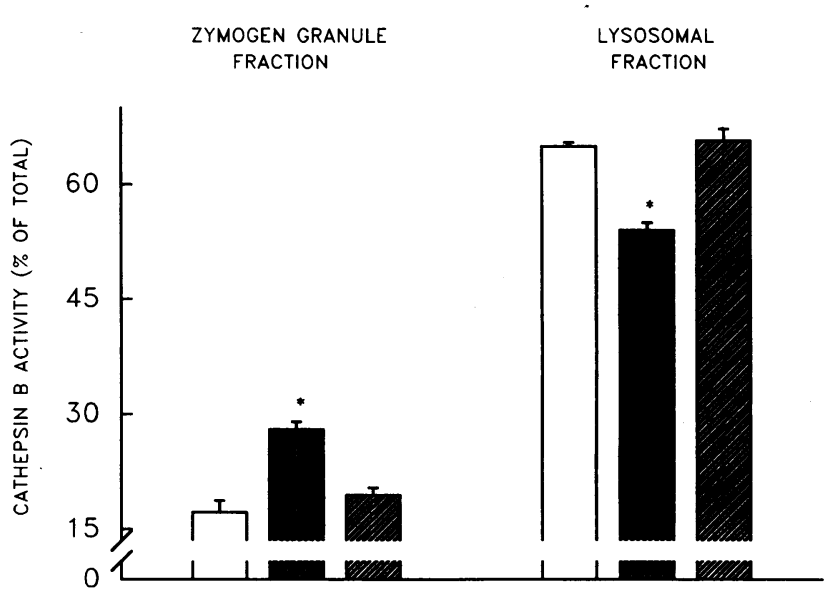

Figure 2. Effect of proteolytic inhibitors on cathepsin B redistribution. Pancreas fragments were incubated in buffer (open bars), plasma obtained from supramaximally stimulated animals with $0.1 \mu \mathrm{M}$ caerulein added ("caerulein-plasma," cross-hatched bars), and caeruleinplasma plus caerulein to which soyabean trypsin inhibitor $(0.1 \mathrm{mg} /$ $\mathrm{ml})$ and phenylmethylsulfonyl fluoride $(1 \mathrm{mM})$ had been added (diagonal bars). After incubation at $37^{\circ} \mathrm{C}$ for $4 \mathrm{~h}$, cathepsin B activity in the zymogen granule and lysosome-enriched fractions was measured. Bars denote means, and vertical brackets denote SEM for results from three separate experiments. ${ }^{*}$ Significant differences $(\alpha$ $\leq 0.01)$ from other groups.

redistribution is dependent upon the presence of protease activity. Using the technique of ultrafiltration, the plasma factor was found to pass through 30,000 but not 10,000 mol wt permeable filters (Table II) suggesting that it has a molecular weight of between 10,000 and 30,000 D.

Incubation of pancreas fragments in caerulein-plasma was found to stimulate amylase secretion (data not shown), an observation which suggested that caerulein-plasma contains significant concentrations of a pancreatic secretagogue most

Table II. Molecular Weight Determination of the Plasma Factor Involved in Redistribution of Cathepsin B

\begin{tabular}{llc}
\hline \multirow{2}{*}{ Group } & \multicolumn{2}{c}{ Cathepsin B activity (\% of total) } \\
\cline { 2 - 3 } & ZG fraction & Lysosome fraction \\
\hline Buffer & $18.5 \pm 0.5$ & $59.5 \pm 1.1$ \\
CER plasma + CER & $31.0 \pm 0.3^{*}$ & $42.5 \pm 0.5^{*}$ \\
10,000 MW filtrate + CER & $18.1 \pm 0.6$ & $62.2 \pm 3.0$ \\
10,000 MW retentate + CER & $29.5 \pm 2.0^{*}$ & $48.0 \pm 3.2^{*}$ \\
30,000 MW filtrate + CER & $34.5 \pm 1.0^{*}$ & $47.0 \pm 1.0^{*}$ \\
30,000 MW retentate + CER & $29.5 \pm 2.0^{*}$ & $49.0 \pm 0.8^{*}$
\end{tabular}

Rat pancreatic fragments were incubated in buffer, caerulein-plasma (see legend for Fig. 1) and caerulein-plasma fractions obtained after ultrafiltration with centiprep-10 (membrane cutoff of $10,000 \mathrm{MW}$ ) and centiprep-30 (membrane cutoff of $30,000 \mathrm{MW}$ ) filters. Caerulein (CER, $0.1 \mu \mathrm{M}$ final concentration) was added to the incubation mixtures as indicated. After $4 \mathrm{~h}$ of incubation at $37^{\circ} \mathrm{C}$, the percentage of total cathepsin B activity recovered in the zymogen granule-enriched and lysosome-enriched fractions was measured. Results shown are means \pm SEMs obtained from three experiments.* Differences which were significant at a level 0.05 or less compared to other groups. 
likely caerulein. To determine the relative importance of the secretagogue caerulein, itself, and the plasma factor(s) expressed in response to in vivo supramaximal stimulation as agents which could elicit in vitro subcellular redistribution of cathepsin B, the potent cholecystokinin receptor antagonist L-364,718 (16) was added to caerulein-plasma. Cathepsin B redistribution did not occur when pancreas fragments were exposed to caerulein-plasma to which L-374,718 had been added (Table I). This observation, combined with the finding that in vitro redistribution of cathepsin B did not occur when fragments were incubated in buffer or normal plasma containing a supramaximally stimulating concentration of caerulein (Fig. 1 ), indicates that in vitro redistribution of cathepsin B results from the combined effects of cholecystokinin receptor occupancy by an agonist and exposure to the plasma factors expressed in response to in vivo supramaximal stimulation.

The time- and concentration-dependent effects of the in vitro cathepsin $B$ redistribution phenomenon are shown in Figs. 3 and 4. Incubation of fragments with caerulein-plasma plus a supramaximally stimulating concentration of caerulein for $<2 \mathrm{~h}$ did not cause significant cathepsin B redistribution. The redistribution phenomenon was clearly evident after $2 \mathrm{~h}$ in vitro incubation and appeared to be complete after $4 \mathrm{~h}$ incubation. The effect of longer in vitro incubation periods could not be evaluated because of poor tissue viability after incubation for longer than $4 \mathrm{~h}$.

Dilution of caerulein-plasma was noted to cause progressive reductions in the magnitude of in vitro cathepsin $B$ redistribution. The use of concentrated caerulein-plasma (three times concentrated using Amicon filters) did not result in greater subcellular cathepsin B redistribution than that noted with unconcentrated caerulein-plasma suggesting that maximal in vitro subcellular redistribution of cathepsin B was induced by exposure to an $80 \%$ (vol/vol) solution of caeruleinplasma plus caerulein.

The above finding suggested that a plasma protease was elaborated during caerulein-induced pancreatitis which could

CATHEPSIN B ACTIVITY IN ZYMOGEN GRANULE FRACTION

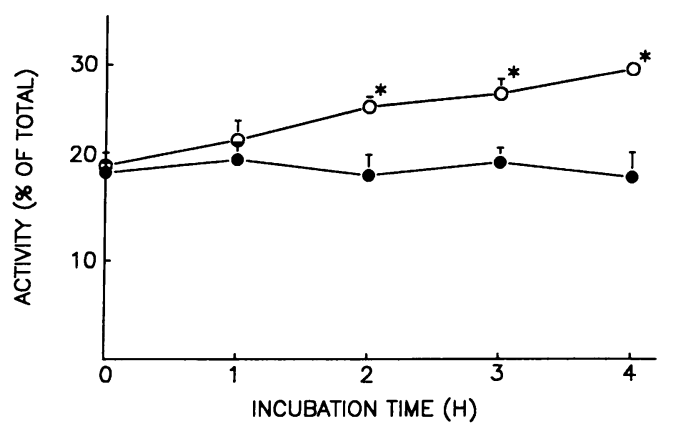

Figure 3. Time-dependence of in vitro cathepsin B redistribution. Pancreas fragments were incubated in buffer with $0.1 \mu \mathrm{M}$ caerulein added (closed circles) or in plasma obtained from supramaximally stimulated animals with $0.1 \mu \mathrm{M}$ caerulein added (open circles) for varying times at $37^{\circ} \mathrm{C}$. After incubation for the indicated time, the fragments were subcellularly fractionated as described in the legend for Fig. 1 and cathepsin B was measured in the zymogen granule fraction. Results shown are mean \pm SEM for three separate experiments. *Significant differences $(P<0.05)$ from values obtained when the fragments were incubated in buffer plus caerulein for that time.
CATHEPSIN B ACTIVITY IN ZYMOGEN GRANULE FRACTION

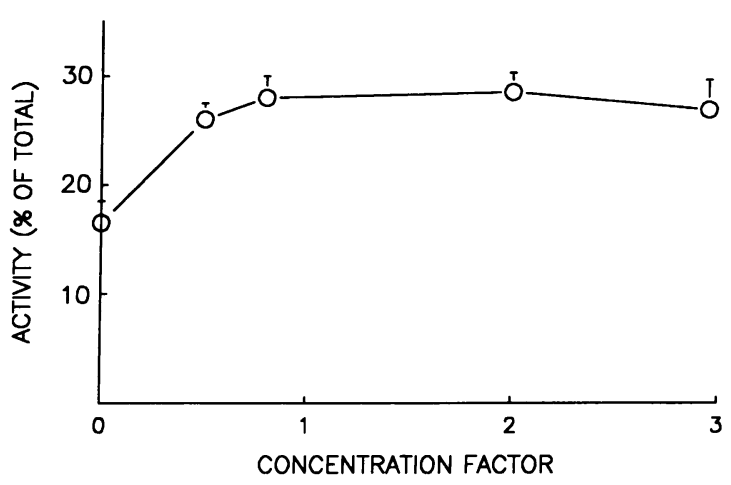

Figure 4. Plasma concentration-dependence for in vitro cathepsin B redistribution. Pancreas fragments were incubated with different concentrations of the plasma obtained from the animals which were supramaximally stimulated with caerulein. In each case, $0.1 \mu \mathrm{M}$ caerulein was added to the plasma. The plasma was concentrated by ultrafiltration with Amicon filters or diluted with Hepes-Ringer buffer. After $4 \mathrm{~h}$ of incubation at $37^{\circ} \mathrm{C}$, the fragments were subcellularly fractionated as described in the legend for Fig. 1, and cathepsin $B$ activity in zymogen granule fraction was quantitated. Results shown are mean \pm SEM from three separate experiments. A concentration factor of 1 is defined as that noted when fragments are suspended in pure plasma.

cause in vitro cathepsin B redistribution. To determine whether a similar phenomenon might be associated with other models of experimental pancreatitis, we evaluated the effects of plasma obtained from opossums after $7 \mathrm{~d}$ of either bile duct ligation ("jaundiced controls") or pancreatic plus bile duct ligation. In accord with the report of Senninger et al. (12), pancreatic-bile duct ligation was found to cause gross and microscopic changes typical of acute necrotizing pancreatitis. As shown in Fig. 5, subcellular redistribution of cathepsin B was observed when rat pancreatic fragments were incubated, in vitro, with caerulein combined with plasma obtained from opossums after pancreatic plus bile duct ligation but not with plasma obtained from the jaundiced control animals that had only bile duct ligation and, as a result, did not have pancreatitis. As had been noted in experiments using rat plasma, the in vitro redistribution of cathepsin B was dependent upon the simultaneous presence of the plasma factor and caerulein stimulation. When the CCK antagonist L-364,718 was added to opossum plasma obtained from jaundiced control animals, $28 \pm 1 \%$ of cathepsin B was recovered in the zymogen granule fraction and $50 \pm 3 \%$ in the lysosome fraction $(n=3)$. When the CCK antagonist was added to opossum plasma obtained from animals with pancreatic duct ligation-induced pancreatitis, $27 \pm 2 \%$ of cathepsin B was recovered in the zymogen granule fraction, whereas $52 \pm 2 \%$ was recovered in the lysosome fraction $(n=3)$. There was, therefore, no significant difference noted when data from pancreatitis and jaundiced control samples plus L-364,718 were compared $(P>0.05)$.

\section{Discussion}

In this communication, we have demonstrated that the subcellular redistribution of cathepsin B which occurs after in vivo exposure to supramaximally stimulating doses of caerulein can 


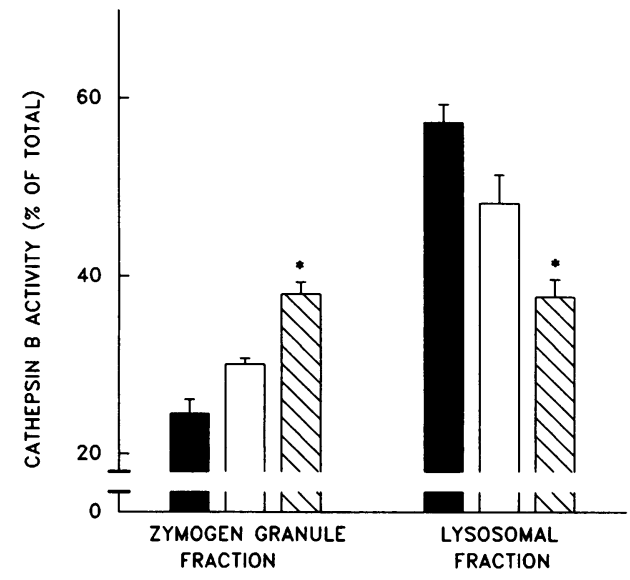

Figure 5. Effects of plasma from duct-obstructed opossums on cathep$\sin B$ distribution. Rat pancreas fragments were incubated for $4 \mathrm{~h}$ at $37^{\circ} \mathrm{C}$ in buffer containing $0.1 \mu \mathrm{M}$ caerulein plus $80 \%$ (vol/vol) plasma obtained from unoperated control opossums (solid bars), jaundiced control opossums that had undergone bile duct ligation $7 \mathrm{~d}$ previously (open bars), or opossums with duct obstruction-induced pancreatitis that had followed pancreatic plus duct ligation (diagonal bars) $7 \mathrm{~d}$ previously. After the fragments were homogenized and subcellularly fractionated, cathepsin B activity in the zymogen granule-enriched and lysosome-enriched fractions was measured and expressed as the percent of total cathepsin B activity. There were at least seven animals in each group. *Values significantly different $(\alpha \leq 0.01)$ from the control unoperated group.

also be induced in vitro. Reproduction of the cathepsin B redistribution phenomenon requires in vitro incubation of pancreas fragments with a supramaximally stimulating dose of caerulein along with plasma taken from a supramaximally stimulated animal. Neither the supramaximally stimulating dose of caerulein nor the plasma from a supramaximally stimulated animal, alone, is sufficient to induce redistribution of cathepsin B. Finally, we have presented evidence which strongly suggests that the factor present in caerulein-plasma which permits cathepsin $B$ redistribution is a protein with a molecular weight between 10,000 and $30,000 \mathrm{D}$ and that its ability to induce in vitro cathepsin B redistribution is dependent upon proteolytic enzyme activity. It is tempting to speculate that the plasma protein is elaborated by in vivo supramaximal stimulation with caerulein although the alternative, i.e., that it is present in an unexpressed or cryptic state before supramaximal stimulation, cannot be excluded. It is also tempting to unify our observations by concluding that the $10,000-30,000$-D plasma protein itself has protease activity. However, other possible explanations must also be considered. For example, the proteolytic activity which is required for in vitro redistribution could be contributed by another plasma factor or even secreted by the acinar cells themselves. In addition, the factor(s) causing in vitro redistribution might do so as a result of its own proteolytic activity or, alternatively, proteolytic hydrolysis of the plasma factor(s) may be required for its activation. It is also possible that the proteolytic events which result in vitro redistribution occur at an intracellular site in response to stimulation or activation by a nonproteolytic plasma factor. At present, it is not possible to discriminate between these various possibilities, but with further purification of the factors involved, these issues should become clarified.
Digestive enzyme zymogens and lysosomal hydrolases such as cathepsin B are synthesized on the rough endoplasmic reticulum and cotransported to the Golgi where the two types of enzymes are segregated from each other $(17,18)$. In previously reported studies, we have shown that in vivo subcellular redistribution of cathepsin B results from a derangement of the normal sorting events as well as from crinophagy (2). The observations reported in this manuscript suggest that a supramaximally stimulating concentration of caerulein when present in vitro along with plasma factor(s) expressed in response to in vivo supramaximal stimulation can also derange the normal sorting events. It is likely that, in this case, caerulein as well as the plasma factor(s) affect intracellular sorting by acting at surface membrane sites but the mechanisms by which intracellular trafficking and sorting are altered remain unknown. It is clear, however, that inhibition of secretion is not, by itself, sufficient to cause redistribution of cathepsin $B$ because digestive enzyme secretion is inhibited by a supramaximally stimulating concentration of caerulein even in the absence of the plasma factor(s). Similarly, interaction of an agonist such as caerulein with low-affinity receptors, a phenomenon recently noted to play a role in secretagogue-induced pancreatitis (19), cannot by itself be the cause of redistribution because low-affinity receptor occupancy by caerulein occurs even in the absence of the plasma factor(s).

Studies aimed at elucidating the events which are critical to intracellular sorting of newly synthesized proteins have been hampered by the relatively few maneuvers which can perturb the sorting process. For the most part, experimental modulation of that phenomenon has involved the use of agents which interfere with transport of lysosomal enzymes to lysosomes by preventing acidification of the lysosomal compartment (20, 21 ). When redistribution of lysosomal hydrolases has been induced by in vivo exposure to a supramaximally stimulating dose of caerulein, lysosomal and digestive enzymes have been found to be colocalized in cytoplasmic vacuoles which have an acidic pH (22). Based on that observation, it would appear unlikely that the in vitro redistribution phenomenon is caused by a failure of lysosomal acidification although studies directly evaluating lysosomal $\mathrm{pH}$ after in vitro redistribution will be needed to settle this issue. If defective lysosomal acidification is not found to underlie in vitro redistribution, the system described in this report may represent a useful alternative method of perturbing intracellular trafficking and, as such, may prove useful for studies probing the events which are critical to that process.

The redistribution phenomenon elicited by in vitro exposure to caerulein plasma along with a supramaximally stimulating concentration of caerulein differs in at least two ways from that which is elicited by in vivo exposure to a supramaximally stimulating dose of caerulein. First, the magnitude of cathepsin $B$ redistribution is significantly less when the phenomenon is elicited in vitro than when it is induced in vivo. For example, after in vivo redistribution $50-60 \%$ of cathepsin B activity can be recovered in the zymogen granule-enriched fraction whereas only $25-35 \%$ of cathepsin B activity is recovered in that subcellular fraction after in vitro redistribution. The second difference involves the relative paucity of morphological changes after in vitro redistribution. We (2-5) and others $(9$, 22) have previously noted the extensive cytoplasmic vacuolization which occurs after in vivo supramaximal stimulation and, indeed, we have noted that digestive zymogens and lysosomal 
hydrolases become colocalized within those vacuoles (3). In contrast, although incubation of pancreas fragments for $4 \mathrm{~h}$ in buffer without caerulein or the plasma factor(s) results in a modest degree of cytoplasmic vacuolization, no increase in vacuolization or colocalization of digestive enzymes with lysosomal hydrolases is noted when a supramaximally stimulating concentration of caerulein and caerulein plasma are present (Meldolesi, J., A. Villa, and M. Lerch, unpublished observation). It is likely that these two differences between the in vivo and in vitro redistribution phenomena are interrelated, i.e., that morphological changes elude detection because the magnitude of redistribution is low. Furthermore, the relatively small magnitude of in vitro redistribution suggests that factors in addition to those in the plasma and a supramaximally stimulating concentration of caerulein may play important roles in determining the magnitude of cathepsin B redistribution.

Subcellular redistribution of lysosomal hydrolases has been noted in three distinctly different experimental models of pancreatitis. In addition to the model involving supramaximal secretagogue stimulation (4), lysosomal enzyme redistribution has been observed after short-term pancreatic duct obstruction (6) and after ingestion of a choline-deficient ethionine-supplemented diet (5). These observations suggested that those models might also involve elaboration of a plasma factor which could induce in vitro cathepsin B redistribution. Studies evaluating this issue in the diet-induced model of pancreatitis are impractical because the ethionine-containing diet only induces hemorrhagic pancreatic necrosis in young (10-15 g) mice (23), and harvesting of sufficient quantities of plasma from these animals would be difficult. Senninger has noted that ligation of the opossum pancreatic and bile duct induces necrotizing pancreatitis. In this report, we note that in vitro subcellular cathepsin B redistribution also occurs when rat pancreatic fragments are incubated with caerulein plus plasma obtained from opossums with pancreatic plus bile duct ligation-induced pancreatitis. On the other hand, in vitro redistribution does not occur when rat pancreatic fragments are incubated with caerulein combined with either normal opossum plasma or plasma obtained from jaundiced control opossums that had undergone only bile duct ligation and did not have pancreatitis. In vitro redistribution was noted only when opossum pancreatitis plasma was combined with caerulein and it was not seen when the CCK antagonist L-364,718 was added to plasma taken from animals with pancreatic duct ligation-induced pancreatitis. These findings indicate that, as with caerulein-induced pancreatitis in rats, the plasma factor which is elaborated can induce in vitro cathepsin $B$ redistribution only when its presence is combined with caerulein stimulation in vitro. Furthermore, these observations suggest that elaboration of a plasma factor capable of inducing in vitro cathepsin $B$ redistribution may be a general feature of other forms of acute pancreatitis as well.

\section{Acknowledgments}

This work is supported by a National Institutes of Health grant \#DK31396. M. M. Lerch is supported by a grant of the Deutsche Forschungsgemeinschaft Nr. Le 625/1-1.

\section{References}

1. Koike, H., M. L. Steer, and J. Meldolesi. 1982. Pancreatic effects of ethionine: blockade of exocytosis, crinophagy and autophagocytosis precede cellular necrosis. Am. J. Physiol. 242:G297-G307.

2. Saito, I., A. Saluja, M. L. Steer, and J. Meldolesi. 1987. Intracellular transport of pancreatic zymogens during caerulein supramaximal stimulation. Am. J. Physiol. 251:G517-G526.

3. Watanabe, O., F. M. Baccino, M. L. Steer, and J. Meldolesi. 1984. Effects of supramaximal caerulein stimulation of the ultrastructure or rat pancreatic acinar cell: early morphological changes during the development of experimental pancreatitis. Am. J. Physiol. 246:G457-G467.

4. Saluja, A. K., S. Hashimoto, M. Saluja, R. E. Powers, J. Meldolesi, and M. L. Steer. 1987. Subcellular redistribution of lysosomal enzymes during caerulein-induced pancreatitis. Am. J. Physiol. 251:G508-G516.

5. Ohshio, G., A. Saluja, U. Leli, A. Sengupta, and M. L. Steer. 1989. Esterase inhibitors prevent lysosomal enzyme redistribution in two non-invasive models of experimental pancreatitis. Gastroenterology. 96:853-859.

6. Saluja, A., M. Saluja, A. Villa, U. Leli, P. Rutledge, J. Meldolesi, and M. L. Steer. 1989. Pancreatic duct obstruction in rabbits causes digestive zymogen and lysosomal enzyme colocalization. J. Clin. Invest. 84:1260-1266.

7. Greenbaum, L. A., and A. Hirshkowitz. 1961. Endogenous cathepsin activates trypsinogen in extracts of dog pancreas. Proc. Soc. Exp. Biol. Med. 107:7476.

8. Steer, M. L., and J. Meldolesi. 1987. The cell biology of experimental pancreatitis. N. Engl. J. Med. 316:144-150.

9. Lampel, M., and H. Kern. 1977. Acute interstitial pancreatitis in the rat induced by excessive doses of a pancreatic secretagogue. Virch. Arch. A Pathol. Anat. Histol. 373:97-117.

10. Saluja, A., I. Saito, M. Saluja, M. J. Houlihan, R. Powers, J. Meldolesi, and M. L. Steer. 1985. In vivo rat pancreatic acinar cell function during supramaximal stimulation with caerulein. Am. J. Physiol. (Gastrointest. Liver Physiol.) 249:G702-G710.

11. Tartakoff, A., and J. E. Jamieson. 1974. Fractionation of guinea pig pancreas. Methods Enzymol. 31:41-59.

12. Senninger, N., F. G. Moody, J. C. U. Coelho, and D. H. Van Buren. 1986. The role of biliary obstruction in the pathogenesis of acute pancretitis in the opossum. Surgery (St. Louis). 99:688-693.

13. McDonald, J. K., and S. Ellis. 1975. On the substrate specificity of cathepsin B1 and B2 including a new fluorogenic substrate for cathepsin $\mathrm{B}_{1}$. Life Sci. 17:1269-1276

14. Tukey, J. W. 1953. The problems of multiple comparisons. Department of Statistics, Princeton University, Princeton, NJ.

15. Zar, J. H. 1984. Multiple comparisons. In Biostatistical Analysis. Prentice Hall, Inc., Englewood Cliffs, NJ. 185-205.

16. Chang, R. S. L., and V. J. Lotti. 1986. Biochemical and pharmacological characterization of an extremely potent and selective nonpeptide cholecystokinin antagonist. Proc. Natl. Acad. Sci. USA. 83:4923-4926.

17. Rosenfield, M. G., G. Kreibich, D. Popov, K. Kato, and D. D. Sabatini. 1982. Biosynthesis of lysosomal hydrolases: their synthesis in bound polysomes and the role of co- and post-translational processing in determining their subcellular distribution. J. Cell Biol. 93:135-143.

18. Sly, W. S., A. Natowicz, A. Gonzalez-Noreiga, J. F. Grubb, and M. D. Fisher. 1981. The role of the mannose-6-phosphate recognition marker and its receptor in the uptake and intracellular transport of lysosomal enzymes. In Lysosomes and Lysosomal Storage Diseases. J. W. Callihan, and J. A. Lowden, editors. Raven Press, New York. 131-145.

19. Saluja, A., M. Saluja, H. Printz, A. Zavertnik, A. Sengupta, and M. L. Steer. 1989. Experimental pancreatitis is mediated by low affinity CCK receptors which inhibit digestive enzyme secretion. Proc. Natl. Acad. Sci. USA. 86:89698971.

20. Cardelli, J. A., J. Richardson, and D. Miears. 1989. Role of acidic intracellular compartments in the biosynthesis of Dictyostelium lysosomal enzymes. The weak bases ammonium chloride and chloroquine diffentially affect proteolytic processing and sorting. J. Biol. Chem. 6:3454-3463.

21. Gonzalez-Noriega, A., J. H. Grubb, V. Talkad, and W. S. Sly. 1980. Chloroquine inhibits lysosomal enzyme pinocytosis and enhances lysosomal enzyme secretion by impairing receptor recycling. J. Cell Biol. 85:839-852.

22. Neiderau, C., and J. H. Grendell. 1988. Intracellular vacuoles in experimental acute pancreatitis in rats and mice are an acidified compartment. J. Clin. Invest. 81:229-236.

23. Lombardi, B., L. W. Estes, and D. S. Longnecker. 1975. Acute hemorrhagic pancreatitis (massive necrosis) with fat necrosis induced in mice by DLethionine fed with a choline-deficient diet. Am. J. Pathol. 79:464-480. 